\title{
Une publicisation entre-soi : quand les patients atteints de cancer prennent la parole
}

\author{
Sandrine KNOBÉ
}

Les années 1990 voient fleurir dans les médias un certain nombre de scandales sanitaires où les victimes elles-mêmes interviennent dans le débat public. Le drame du sang contaminé, en particulier, a fortement marqué l'opinion publique ainsi que les acteurs politiques eux-mêmes, dans la mise en cause de responsabilités au plus haut niveau de l'État (Fillion, 2009). D'autres affaires vont tour à tour connaître une publicisation importante, portées ou non par des collectifs porte-parole de victimes, parmi lesquelles les affaires relatives à l'hormone de croissance (1992), à l'amiante (1995), à la vache folle (1996) ou à la pollution de La Hague (1997) par exemple. La multiplication de ces "affaires » mais surtout leur médiatisation et la mise en exergue d'usagers-victimes participent à créer des conditions sociales favorables à la naissance de mouvements contestataires portés par les usagers eux-mêmes, ou par des collectifs parlant en leur nom. Ils parviennent à diffuser auprès d'un très large public, bien au-delà des seules personnes concernées ou susceptibles de l'être, leurs revendications visant au déploiement, en termes de santé publique, de mesures spécifiques de réparation et de prévention. La thématique de la participation des usagers au système de soins s'insère ainsi dans le débat politique et connaît plusieurs traductions juridiques, jusqu'à devenir un enjeu reconnu sous l'appellation de "démocratie sanitaire » aboutissant à la promulgation de la loi $\mathrm{n}^{\circ} 2002-303$ relative «aux droits des malades et à la qualité du système de soin », adoptée le 4 mars 2002.

$\mathrm{Au}$ cours de cette période, les premiers «États généraux des malades atteints de cancer » (La Ligue, 1999) qui rassemblent environ 1300 malades, sont organisés par la Ligue nationale contre le cancer à Paris en novembre 1998. L'événement sera perçu comme un acte fondateur de la prise de parole des malades par les acteurs de la lutte contre le cancer (Azzedine et al., 2007 ; Castel, 2005). C'est aussi à la fin des années 1990 que vont être créées deux associations de lutte contre le cancer se positionnant clairement du côté de l'activisme politique en faveur des patients. Il s'agit de l'association Europa Donna - Forum France (EDFF) (encadré 1) qui tient à constituer un groupe de pression politique spécifiquement dédié à la lutte contre le cancer du sein, et de Jeunes Solidarité Cancer (JSC) (encadré 1) qui oriente ses actions de lobbying en direction des adolescents et des jeunes adultes atteints de cancer.

Nous avons procédé, auprès de ces deux associations, à des enquêtes de terrain distinctes dont nous reprenons ici les données empiriques afin d'apporter des éléments de réflexion sur l'articulation entre les domaines sanitaires privés et publics, en mettant plus particulièrement en évidence les difficultés d'une montée en généralité d'histoires singulières. En ce qui concerne "Europa Donna - Forum France », notre étude porte spécifiquement sur une délégation locale (DL) où nous avons réalisé des observations au cours de réunions mensuelles entre avril 2006 et octobre 2007. D'une durée moyenne de trois heures, ces réunions rassemblent entre quinze et vingt femmes qui représentent les membres actifs. Nous avons également procédé au recueil d'entretiens semi-directifs avec seize participantes à ces réunions mensuelles ainsi qu'avec deux membres du conseil d'administration de l'association (au niveau national). Parmi les dix-huit personnes interviewées, quatorze sont des patientes ou d'anciennes patientes atteintes d'un cancer du sein. Ayant spécifiquement pour objet la lutte contre le cancer du sein, les membres de l'association sont exclusivement des femmes. De manière générale, elles 
sont plus nombreuses que les hommes à s'engager dans les associations de lutte contre le cancer (Ferrand-Bechmann, 2011). Il en est de même pour "Jeunes Solidarité Cancer », qui ne compte que $10 \%$ d'hommes parmi ses membres alors même que ses actions ne sont pas focalisées sur un cancer typiquement féminin. Pour cette seconde association, notre analyse porte sur les contenus du forum Internet pour la période 2010-2012. Parmi les 100 utilisateurs répertoriés durant cette période, 66 ont indiqué leur âge qui s'élève en moyenne à 26 ans. La tranche d'âge cible de l'association n'est pas clairement délimitée, le terme «jeunes» s'appliquant aussi bien à des adolescents, mineurs, qu'à de jeunes adultes voire à des patients plus âgés (l'utilisateur le plus âgé du forum a 49 ans). Est « jeune » celui qui se considère comme tel, comme nous l'a expliqué le président de l'association. Nous avons également complété notre enquête par une dizaine d'entretiens semi-directifs réalisés en face-à-face avec des usagers de ce forum et des administrateurs de l'association.

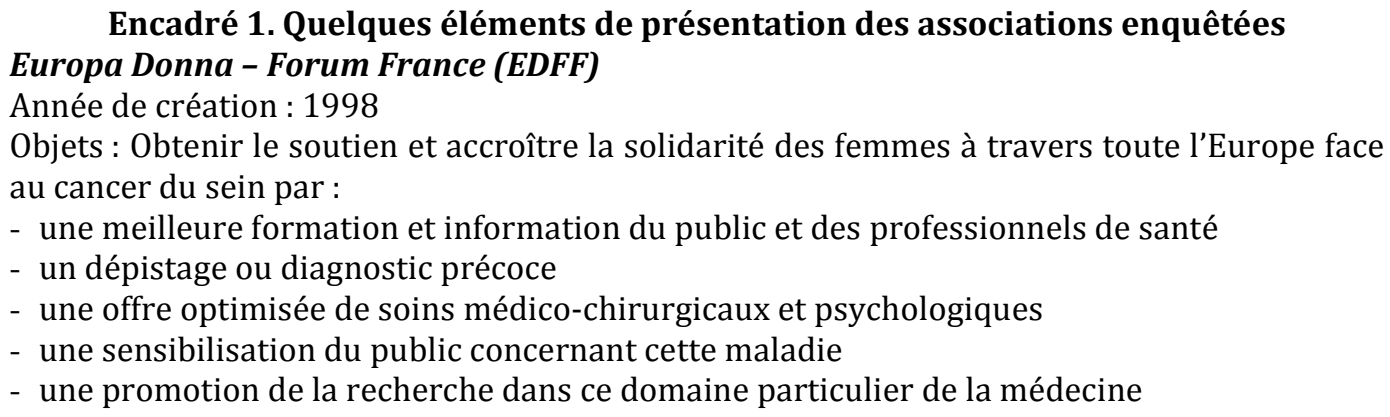

\section{Jeunes Solidarité Cancer (JSC)}

Année de création : 2002

Objets : Briser l'isolement des jeunes adultes malades, anciens malades et de leurs proches par :

- le soutien par le forum de discussion sur Internet

- l'information par le site Internet, une série de guides et un carnet de bord

- la lutte contre la précarité socioprofessionnelle par un travail de fond en collaboration avec de nombreuses associations.

Sources : sites Internet des deux associations, www.europadonna.fr et www.jeunessolidaritecancer.org

Si ces deux associations se positionnent dans le registre de l'activisme politique et publicisent régulièrement des revendications relatives aux intérêts spécifiques des patients qu'elles représentent, comment les expériences personnelles de la maladie sont-elles évoquées publicisées entre les patients eux-mêmes? Le passage d'une expression privée à une publicisation collective va-t-il de soi ? Nous souhaiterions montrer qu'il est loin d'aller de soi et qu'il se heurte à un "entre-soi » des patients difficile à dépasser. L'expérience personnelle de la maladie ne se transforme en cause collective qu'à la suite d'un travail associatif dont les principaux acteurs ne sont pas nécessairement les patients eux-mêmes. Pourquoi? Deux aspects seront plus particulièrement discutés ici, prenant en compte les attentes sociales en termes de prise de parole, notamment au début de la trajectoire de la maladie du cancer (Ménoret, 1999 D'une part, la recherche principale d'une entraide entre patients et d'autre part, la difficulté à effectuer une montée en généralité à partir des expériences singulières des patients, compromettent le passage du privé au public car, est empêchée de la sorte, la construction d'un nous collectif porteur de revendications transversales. En l'espace de quelques semaines souvent, le temps de réaliser les examens nécessaires et d'en attendre les résultats, un diagnostic de cancer fait passer un corps, parfois 
asymptomatique, du statut de sain à celui de malade. L'entrée dans ce nouveau statut social de malade, mais aussi le caractère potentiellement létal de cette maladie, la complexité des traitements et la lourdeur de leurs effets secondaires, incitent certains patients, par le biais d'une association notamment, à rencontrer d'autres patients ou d'anciens patients afin de partager leurs expériences communes et singulières. Ainsi, en début de trajectoire ${ }^{1}$ ou en cas de récidive, un régime de proximité directe avec la maladie, du fait d'être soi-même malade et souvent alors en cours de traitement médical, encourage les attentes en termes d'entraide entre patients et/ou avec d'anciens patients. Cette proximité semble surtout valoriser une prise de parole individuelle soulignant la particularité de chaque expérience. Ces deux aspects, entraide et mise en avant de la singularité des parcours, rendent difficile une publicisation collective car les patients, même engagés dans des associations à vocation militante, restent centrés sur leurs propres expériences. Le passage du privé au public n'est ainsi pas en mesure de se réaliser ou, s'il s'effectue, c'est par l'intermédiaire d'une délégation de parole à des personnes non-malades pour ce qui concerne les deux associations dont il est question ici.

\section{Prendre la parole pour s'entraider}

La DL d'EDFF autorise un engagement associatif dès l'entrée dans la maladie et l'encourage même dans la mesure où sa création était liée au sentiment d'absence de lieu d'information et d'échanges entre patientes. D'autres associations, comme par exemple «Vivre comme avant» dont l'activité principale consiste, au sein des établissements de soins, dans la visite, par d'anciennes patientes, de patientes opérées ou traitées pour un cancer du sein, exigent de leurs bénévoles (ayant été atteintes de cancer) un temps de recul d'avec la fin de leurs traitements, de l'ordre en général de deux à trois ans minimum. Pour des personnes au début de leur parcours de maladie, la DL enquêtée constitue la seule possibilité d'un engagement associatif local en tant que bénévole et non pas seulement bénéficiaire. JSC, de la même manière, grâce à son forum Internet, permet aux adolescents et jeunes adultes de s'exprimer dès l'annonce de leur maladie.

Ainsi, la DL compte presque exclusivement des patientes ou d'anciennes patientes, du moins parmi ses membres actifs ${ }^{2}$, et souvent ce sont des patientes proches du début de leur trajectoire de la maladie ${ }^{3}$. Les activités proposées par la DL sont essentiellement orientées vers l'acquisition et la diffusion d'informations sur des sujets relatifs au cancer du sein ou plus généralement à la maladie. La possibilité d'obtenir des réponses précises à des questions d'ordre médical grâce à la présence, aux réunions, d'un référent médical, semble permettre aux patientes de clarifier leur compréhension des traitements. Il est également possible de solliciter un entretien individuel avec ce référent médical. De plus, le partage d'expériences personnelles entre les différents membres, par l'échange

\footnotetext{
${ }^{1}$ Comme le confirment les données issues des entretiens réalisés.

${ }^{2}$ Sont désignés comme tels les membres de la DL qui se rendent régulièrement aux réunions mensuelles (par différenciation des membres uniquement adhérents, c'est-à-dire ayant acquitté leur cotisation à l'association).

${ }^{3}$ Parmi les quatorze patientes interviewées, seules deux ont pris connaissance de leur diagnostic de cancer du sein (ou de leur récidive) il y a plus de trois ans.
} 
notamment des «bonnes» adresses de divers praticiens (kinésithérapeute, homéopathe, chirurgien...), est particulièrement apprécié par les patientes dont le cancer vient d'être diagnostiqué. Cette double proximité directe avec la maladie, dans la mesure où les personnes participant aux réunions mensuelles de la DL sont ou ont été ellesmêmes malades et que souvent elles sont encore en traitement quand elles rejoignent la DL, transparaît dans les motifs d'engagement qu'elles expriment.

P., qui a pris contact avec la DL un mois après l'annonce du diagnostic de son cancer en 2005, explicite ainsi ses attentes associatives: Quand on vous annonce un cancer, on est complètement perdu, on a besoin d'informations, on a besoin de savoir, on a besoin de connaissances qu'on n'a pas. Donc, je me suis dit super, il y a une association qui va pouvoir m'accueillir et répondre à mes questions. [...] Avoir de l'information. Obtenir aussi des noms de médecins pour les médecines parallèles qu'on ne connaît pas comme ça d'emblée. Donc on m'a indiqué un homéopathe [...] et ça, ça m’a aussi beaucoup aidé [...] Qu'on réponde à mes questions aussi (48 ans, employée, diagnostic de cancer du sein en 2005, membre de la DL depuis 2005).

Les activités proposées par la DL mettent l'accent sur la singularité des expériences par l'expression des parcours et des ressentis personnels. Cela est certes le cas au cours des séances du groupe de parole mais aussi lors du traditionnel «tour de table » où chaque participant à la réunion de la DL se présente, ou plutôt présente brièvement son parcours de maladie, et ceci lorsqu'il y a de nouvelles personnes qui assistent à la réunion mensuelle. Cette réunion constitue de la sorte un véritable moment « entre soi » (Barbot, 1995) que le rappel fréquent d'une certaine convention de confidentialité ne manque pas d'accentuer.

Ce que je voudrais dire c'est qu'ici on a effectivement aussi des règles de confidentialité. On aimerait que ce qui est personnel aux autres ne sorte pas d'ici. Bien sûr on peut raconter ce qu'on a fait, on peut apprendre des choses et en parler, c'est tout à fait normal, mais ce qui est personnel ça, ça reste confidentiel [journal de terrain, octobre 2006] ${ }^{4}$.

Cette proximité transparaît également chez les utilisateurs du forum Internet de JSC. Ainsi, parmi les cent utilisateurs du forum recensés entre juin 2010 et juin 2012, quasiment les trois quarts sont des patients en cours de traitement ou d'anciens patients.

Figure 1. Statut des utilisateurs du forum Internet de JSC

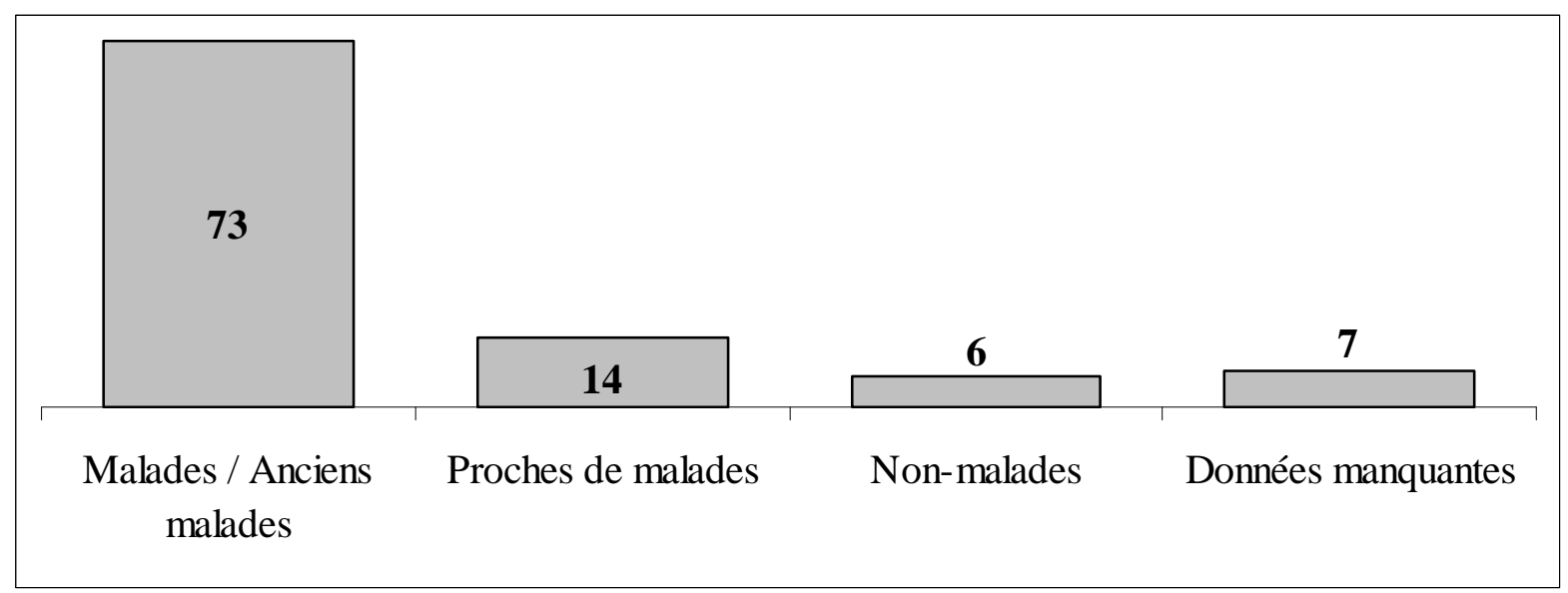

Source : analyse des contenus du forum Internet de JSC pour la période 2010-2012 avec catégorisation des utilisateurs en fonction des indications données par eux-mêmes sur leur statut.

\footnotetext{
${ }^{4}$ Un tel rappel a été effectué au cours de huit des seize réunions auxquelles j’ai assisté.
} 
Et, comme pour la DL d'EDFF, la présentation de son propre parcours revêt un caractère très important dans la dynamique du forum comme en atteste l'intitulé de la rubrique: «STOP! Le petit passage obligatoire ${ }^{5}$ tirez-nous votre portrait! ». Ainsi, les nouveaux venus sur le forum postent généralement un premier message dans cette rubrique, détaillant plus ou moins leur parcours de maladie et insistant sur le partage d'une expérience commune.

Bonjour, je suis toute nouvelle sur le forum. J'ai 28 ans et on vient de me diagnostiquer un deuxième cancer du sein... Et oui, après 1 an et demi (en enfer) a soigné mon sein droit, il y a 10 jours on a trouvé un nodule dans mon sein gauche qui s'est avéré cancéreux! Enfin je suis plutôt bien entourée, il me manque juste l'échange avec des gens de mon âge ayant vécu la même chose, HELP ! (message posté en octobre 2011).

Salut à tous, je m'appelle F., j'ai 22 ans. On m'a découvert un cancer rare de la plèvre suite à un pleurotalcage de la plèvre en septembre dernier. Je viens d'avoir mon premier traitement sur Paris il y a quelques jours. Je découvre le monde de cette maladie petit à petit. Sinon dans la vie, comble du mauvais sort, je suis actuellement étudiante infirmière en deuxième année, pour le moment je n'ai pas eu le courage de stopper mes études même si j'ai beaucoup de mal à suivre les cours mais c'est pour moi essentiel de continuer au maximum la vie normale. Je suis très ravie de voir que finalement je ne suis pas seule à être dans la même galère et ça me redonne le moral. On va se soutenir et on va y arriver ! (message posté en janvier 2012).

L'importance de cette rubrique, de ce passage obligé légitimant en quelque sorte sa présence sur le forum et signifiant l'appartenance à la " classe des malades ${ }^{6}$, s'exprime également par le nombre de messages postés dans les rubriques à caractère plus personnel7.

\section{Figure 2. Nombre de messages dans les différentes rubriques du forum}

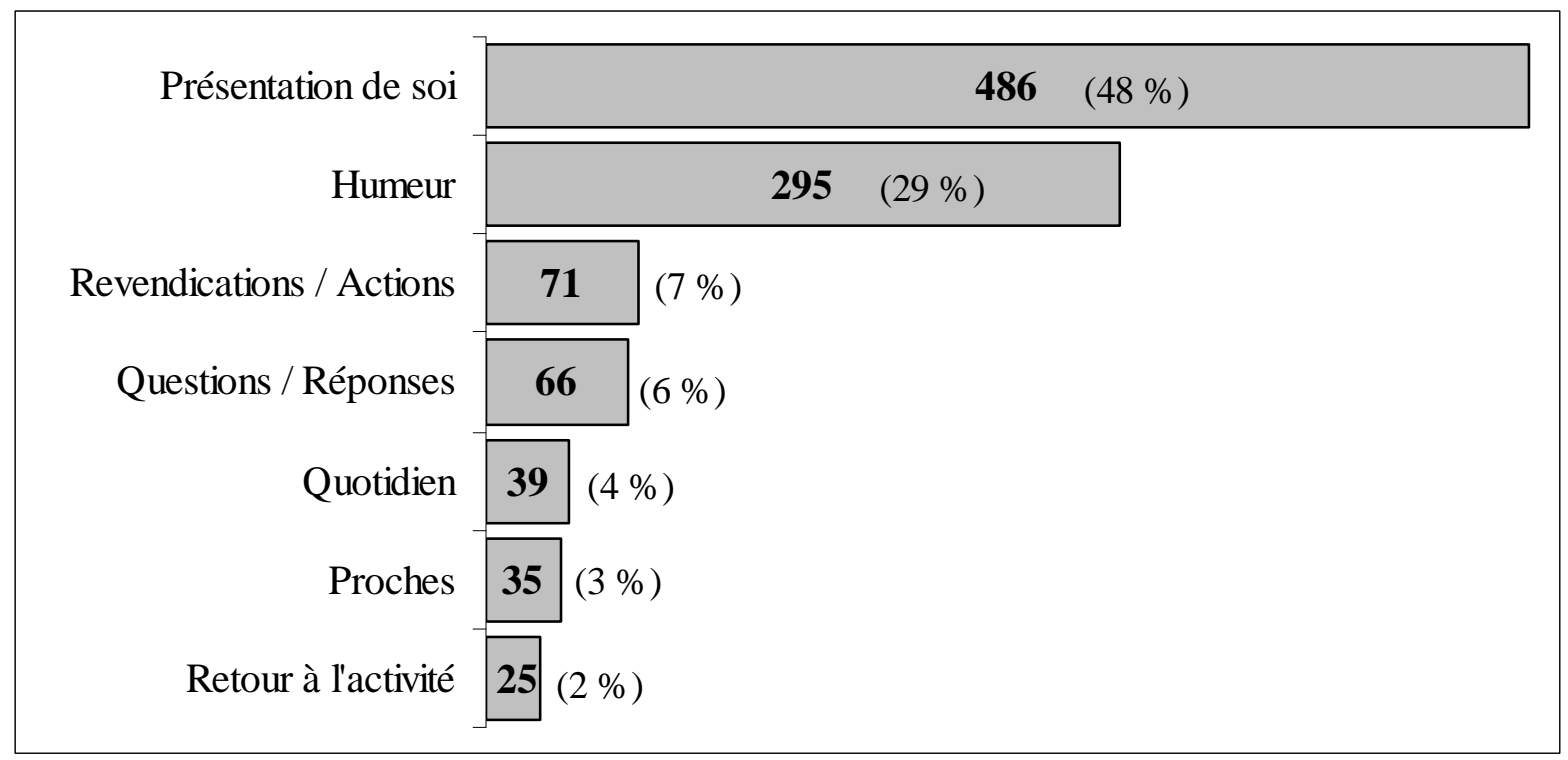

\footnotetext{
5 Souligné par nous.

6 Pour Dan Ferrand-Bechmann (2011: 42), l'appartenance à cette «classe des malades » implique que «les participants s'y sentent épaulés et inclus dans le confort d'une communauté de sentiments, d'émotions et de souvenirs ».

${ }^{7}$ Il en serait de même des blogs dans le domaine du cancer où seuls $9 \%$ de ceux recensés développent des thèmes relatifs aux politiques de santé (Legros, 2009).
} 
Source : analyse des contenus du forum Internet de JSC pour la période 2010-2012 avec décompte du nombre de messages dans chaque rubrique que compte le forum.

Ce mode de fonctionnement permet certes aux patients et aux anciens patients d'aborder des aspects très intimes liés à leurs traitements ou à leurs angoisses (Akrich, Méadel, 2009 ; Kivits, 2012). En revanche, la montée en généralité est souvent absente, renforcée par l'idée que "chaque cas est unique ». Il est par conséquent difficile de procéder à des généralisations susceptibles de constituer une cause pouvant faire l'objet d'une mobilisation collective. Faire face, de la sorte, à sa propre maladie ne semble pas favoriser un travail de désingularisation des expériences nécessaire à la montée en généralité et à la formulation de revendications portées au nom d'un collectif (Herzlich, 1995).

\section{Déléguer la parole pour revendiquer}

Ainsi, au sein de la DL d'EDFF, ce travail de désingularisation semble être freiné par la nature de certaines revendications formulées par les membres de la DL eux-mêmes. En effet, ces revendications prennent un caractère plus individuel que collectif car elles sont portées en nom propre et non pas au titre d'un engagement associatif. Le passage d'une formulation privée à une formulation publique n'est pas délégué à un collectif mais s'appuie sur un témoignage personnel. La généralisation et l'extrapolation sur lesquels s'adosse tout travail de construction d'une cause paraissent alors d'autant plus difficiles à mettre en œuvre. Une patiente, membre de la DL, a par exemple été à l'origine de la publication dans un journal d'informations régionales d'un article concernant sa demande d'autorisation de vacances alors qu'elle était en congé de maladie. Cette demande lui a été refusée par la Caisse primaire d'assurance maladie auprès de qui le patient doit adresser une demande officielle s'il souhaite quitter le département pendant son arrêt de travail. Elle a obtenu gain de cause lorsque son médecin traitant à préciser que cette autorisation de vacances devait lui être accordée pour raison de « dépression » (et non pas seulement de cancer).

\section{Encadré 2. Notes de terrain d'observation. Réunion mensuelle de la DL, août 2006.}

V. prend la parole pour expliquer son interpellation des médias locaux :

_Moi, je me suis fâchée cet été. Donc j'ai rencontré un journaliste qui a fait cet article dans le journal. (50 ans, profession intermédiaire, diagnostic de cancer du sein en 2004, membre de la DL depuis 2005)

_Ah ouais, c'était toi ! C'est affiché dans tous les hôpitaux, partout. (Ro, coordinatrice de la DL) [...]

V. raconte ce qui a motivé cet article :

_J'ai explosé, on [la Caisse Primaire d'Assurance Maladie] m'a refusé de partir en vacances...

_Ah bon ! (la salle)

_[Une des personnes de la caisse primaire d'assurance maladie] m'a dit 'vous savez, il faudrait que ça soit marqué, vous êtes dépressive, alors vous avez le droit'. J'ai pris mon papier, je suis retournée chez le médecin, je lui ai posé le papier, il m'a dit 'ben je vais marquer que tu es dépressive (comme c'est mon cousin), tu es dépressive après ta maladie'. J'ai ramené ce papier, j'ai eu le droit de partir. Non mais c'est nul! (V)

Si l'identité personnelle est davantage mobilisée que l'identité collective comme cela a pu être constaté pour d'autres formes de mobilisation (Ion,1997), la DL présente de 
plus la particularité de procéder de manière plus ou moins explicite à des différenciations rendant plus difficiles les réflexions et les prises de position au nom d'un "nous » unificateur. Une première distinction s'établit entre le référent médical et les autres membres, qui s'apparente essentiellement à une différenciation entre malades et non-malades. En effet, la personne au statut de référent médical n'a pas été atteinte d'un cancer du sein, ce qu'elle-même ou les autres ne manquent pas de rappeler à différentes occasions. La définition même du collectif est par conséquent problématique puisque le référent médical par exemple ne s'y inclut pas nécessairement. De nombreuses discussions montrent également les difficultés à circonscrire précisément le groupe en incluant ou en excluant notamment les membres adhérents mais non «actifs » (c'est-à-dire ne participant pas aux réunions mensuelles). Cela empêche la délégation de parole à un collectif car la question de savoir quel groupe il s'agit de représenter reste en suspens : est-ce un collectif de patientes atteintes de cancer du sein ou un collectif de femmes ou plus généralement encore de personnes sensibilisées à cette pathologie ${ }^{8}$ ? La politisation des discours est alors freinée dans la mesure où un collectif conflictuel ${ }^{9}$ n'est pas clairement défini comme tel.

JSC aussi se positionne comme une association militant pour l'amélioration de la prise en charge spécifique des adolescents et jeunes adultes atteints de cancer. Mais, comme le montre le nombre de messages postés dans les différentes rubriques proposées sur son forum Internet, les messages à caractère revendicatif ou proposant des actions concrètes n'arrivent qu'en troisième position loin derrière tout ce qui a trait au partage de l'expérience personnelle de la maladie. Et tout comme pour la DL d'EDFF, on constate une oscillation entre préoccupations individuelles et requête collective. L'exemple suivant constitue un extrait d'une succession de messages postés avec le titre, des aides financières pour les jeunes malades.

Message initial posté en juin 2010 : Déjà qu'avoir un cancer alors qu'on n'a même pas 25 ans c'est pas simple, je trouve qu'il serait bien que les jeunes malades puissent avoir des aides pour conserver leur indépendance. C'est pas forcément facile de retourner chez papa maman et comme à moins de 25 ans, il n'y a même pas de RMI / RSA... Bref, il faudrait penser à leur filer un coup de main à ces jeunes qui en bavent déjà pas mal! Je compte sur vous pour faire passer le mot à Roselyne [Bachelot] ou à je ne sais qui !

Une réponse en juillet 2010 : Bonjour, en effet, depuis l'existence de JSC c'est-à-dire depuis 2000, on
fait remonter ce problème crucial chez les jeunes adultes malades qui se retrouvent sans ressources
car ils n'ont pas assez travaillé voire jamais travaillé pour pouvoir prétendre à des Indemnités
journalières de la Sécurité Sociale. Ils ne peuvent pas prétendre au RSA car âgés de moins de 25 ans
et sans enfant à charge (...) Suivant votre état de santé, vous pouvez demander l'Allocation aux
Adultes Handicapés (AAH) à la Maison Départementale des Personnes Handicapées (MDPH) mais
cette première demande peut prendre 6 à 9 mois avant d'être étudiée. Que faire alors quand on a
rien pour survivre? Faire appel aux aides sociales légales et extra légales, pour cela je vous invite
déjà à faire part de votre situation financière au service social de l'hôpital qui est un maillon
important entre les diverses autres administrations et associations qui pourraient intervenir. Vous
pouvez aussi de vous-même interpeller le service social de la sécurité sociale de votre commune et
département (ponctuellement des aides peuvent vous êtes attribuées pour le paiement d'un loyer,
la prise en charge d'une facture EDF ou GDF...). Les associations de lutte contre le cancer et d'aide
comme la Ligue contre le cancer peuvent aussi intervenir. À voir aussi auprès de sa mutuelle quand
on a la chance d'en avoir une. Si vous voulez des précisions sur tout ça n'hésitez pas, je suis moi

${ }^{8}$ Dans le cas de la lutte contre le sida, Janine Barbot (2002) a pu montrer comment l'identification publique d'une association relevait d'un choix stratégique dans la construction d'une cause, comme par exemple la mise en avant d'une séropositivité politique ou d'une séropositivité biologique.

${ }^{9}$ La politisation des discours (Duschesne, Haegel, 2001 ; 2004) s'opère lorsqu'il y a montée en généralité et reconnaissance par les acteurs de la dimension potentiellement conflictuelle de leur prise de position. 
aussi ancienne malade ${ }^{10}$ et en plus assistante sociale, j'ai connu la précarité pendant la maladie et là aussi je remercie mes parents de m'avoir aidé car c'est pas tous les jours simple de manger à sa faim quand on a rien sur son compte bancaire c'est pour cela qu'il ne faut pas avoir honte de pousser les portes pour avoir des aides. (B. L., Référente Précarité à JSC ${ }^{11}$ )

Une autre réponse en décembre 2010 : On y travaille! Avec JSC, nous faisons partie d'un collectif appelé Chroniques Associés. Il réunit 8 associations autour des maladies chroniques (l'association AIDES, l'Association Française des Diabétiques (AFD), l'Association Française des Sclérosés en Plaques (AFSEP)...). En nous réunissant, nous sommes plus forts pour faire passer des revendications communes à toutes les pathologies (SIDA, cancer, sclérose en plaques, diabète...). Un site, créé par le collectif, sortira en avril. Il sera sous la forme d'un espace communautaire où l'on pourra échanger nos tuyaux, nos coups de gueules, nos idées... J'espère vous y retrouver !

Le message initial est formulé de manière générique et fait part d'une revendication concernant l'ensemble des jeunes atteints de cancer et encourage clairement l'association JSC à la porter à un niveau politique décisionnel. Par contre, la première réponse est caractéristique de ce positionnement ambivalent entre une réponse spécifique au cas individuel et une tentative de montée en généralité. Le rappel du partage de l'expérience de la maladie est également présent. À l'inverse, la deuxième réponse (celle datant de décembre 2010), formulée non pas par une ancienne malade, mais par une proche de malade occupant également une fonction de chargée de mission salariée au sein de JSC à cette époque-là, s'appuie sur un autre registre d'expression. Il s'agit bien de dépasser le cas particulier pour évoquer les problèmes posés par le cancer et plus largement les maladies chroniques en termes généralistes. Une place primordiale est toutefois toujours attribuée aux témoignages de patients. Mais souvent, ils témoignent et ne portent pas eux-mêmes les revendications en lien avec les problèmes qu'ils soulèvent de cette manière, à l'instar de la médiatisation et du mandat opérés par la Ligue nationale contre le cancer au sujet de la prise de parole des patients atteints de cancer par l'intermédiaire des États généraux (Knobé, Briatte, 2010). Ainsi, à EDFF, plus qu'à JSC, ce sont les membres du conseil d'administration, non-malades, qui portent la parole des patientes atteintes de cancer du sein. Et JSC, dont une grande part du travail militant était effectuée par l'un des présidents et fondateurs de l'association, semble vouloir confier à d'autres organisations le mandat revendicatif et institutionnel.

C'est vrai que de plus en plus l'association va s'orienter vers des projets plus directs pour le patient (...) Il y a eu la création de GoAJA [Groupe d'Oncologie Adolescents et Jeunes Adultes], c'est la société savante qui va s'occuper des adolescents et jeunes adultes atteints de cancer. C'est vraiment une équipe pluridisciplinaire, oncologues adultes et oncologues pédiatriques avec aussi les métiers plus dans l'ombre, les métiers paramédicaux et les associations. Ça y est enfin on va travailler ensemble pour la même cause. Et on espère que eux vont prendre la partie institutionnelle et que l'association [JSC] va pouvoir continuer maintenant à travailler vraiment pour proposer des activités directes avec le patient. On estime que maintenant ça fait 10 ans qu'on a travaillé sur la partie institutionnelle, on est reconnu, on est écouté et on nous connaît. Donc ça y est, on va pouvoir s'attaquer plus à des choses concrètes pour les patients. Voilà nos objectifs pour l'association dans les prochaines années même si on va continuer à faire de l'institutionnel et à être présent et à faire avancer la prise en charge mais on le fera à travers GoAJA, pas à travers l'association je pense (bénévole à JSC depuis 2009, proche de malade).

\footnotetext{
10 Souligné par nous.

${ }^{11}$ Il s'agit d'une personne qui au sein de l'association JSC s'occupe plus particulièrement des questions relatives aux situations de précarité que peuvent connaitre les patients atteints de cancer ;
} 
Le passage du privé au public ne semble pas aller de soi, sans doute par la difficulté que représente la montée en généralité des expériences singulières des personnes atteintes par la maladie. L'adhésion à une association de lutte contre le cancer, se positionnant de plus comme militante ${ }^{12}$, ne paraît pas suffire à créer les conditions d'une publicisation d'enjeux de santé spécifiques à une pathologie particulière ou plus transversaux en termes de santé publique. L'association JSC opte alors pour une forme de délégation de cette publicisation en adhérant à d'autres collectifs (comme GoAJA). Les malades (ou anciens malades) y sont représentés tout en étant associés également à des professionnels de santé. Ces derniers garantissent probablement une montée en généralité dissociée de la mise en avant de cas particuliers liée, pour les patients, à leur propre vécu. Il en va de même pour EDFF où les administrateurs, donc les interlocuteurs légitimes des diverses institutions ou représentants politiques ne sont pas d'anciens malades. C'est donc essentiellement la figure d'un malade représenté qui prédomine dans la lutte contre le cancer plutôt que celle d'un malade militant (Knobé, 2009).

\section{Conclusion}

Lorsque les patients ou anciens patients atteints de cancer prennent la parole, il s'agit la plupart du temps, comme nous l'avons vu à travers l'exemple de deux associations à vocation militante, d'une prise de parole entre-soi relevant davantage du partage d'expériences. Ils parlent de leur maladie, de leur parcours, de leurs problèmes, de leurs satisfactions et insatisfactions dans un cadre circonscrit aux réunions de l'association (pour la DL) ou au forum (pour JSC). Le régime de proximité directe avec la maladie, c'est-à-dire le fait d'être soi-même malade et souvent encore en cours de traitement lors de l'engagement associatif, renforce la valorisation de l'entraide et du partage d'expériences. La trajectoire de la maladie (Ménoret, 1999) influe sans conteste sur les attentes relatives à l'engagement associatif (Knobé, 2007 ; Ferrand-Bechmann, 2011). Si dans un premier temps, la proximité avec la maladie semble freiner une forme publique de publicisation, une certaine prise de distance en cas de trajectoire ascendante paraît la décourager à son tour. Les membres de JSC interviewés insistent par exemple sur le turn-over important des bénévoles au sein de l'association, les jeunes abandonnant leur engagement lorsque, en rémission, ils tournent la page de la maladie. Cette difficulté de conciliation entre intérêts personnels et cause collective apporte plus globalement des éléments d'explication quant à la manière dont la représentation des usagers de la santé s'est institutionnalisée en France au cours des années 1990 notamment par le travail militant effectué par le Collectif interassociatif sur la santé (CISS) (Knobé, 2010). En effet, la question de la prise en compte de la parole des usagers de la santé a été construite comme un problème social et politique nécessitant une intervention des pouvoirs publics. L'efficacité de cette publicisation tient à l'alliance des plus grandes associations françaises dans le domaine des luttes contre les maladies (comme par exemple, La ligue nationale contre le cancer, AIDES, l'association française contre les myopathies, etc.) sur des thématiques transversales de santé publique. La montée en généralité a consisté pour le CISS a dépassé les intérêts particuliers liés à chaque

\footnotetext{
12 Dans le cas de la lutte contre le sida, Janine Barbot (2002) a pu montrer comment l'identification publique d'une association relevait d'un choix stratégique dans la construction d'une cause, comme par exemple la mise en avant d'une séropositivité politique ou d'une séropositivité biologique.
} 
pathologie spécifique pour penser et revendiquer des enjeux plus globaux et transversaux (relatifs aux droits des malades ou à l'accès aux soins par exemple).

\section{Références bibliographiques}

Akrich M., Méadel C. (2009), «Les échanges entre patients sur l'internet», La presse médicale, $\mathrm{n}^{\circ} 38$, p. 1484-1493.

Azzedine L., Blanchard G., Poncin C. (2007), «Le cancer dans la presse écrite d'information générale », Questions de communication, ${ }^{\circ} 11$, p. 111-127.

Barbot J., (2002), Les malades en mouvements. La médecine et la science à l'épreuve du sida, Paris, Balland.

Duchesne S., Haegel F. (2001), « Entretiens dans la cité, ou comment la parole se politise », EspacesTemps, n76/77, p. 95-109.

- (2004), « La politisation des discussions, au croisement des logiques de spécialisation et de conflictualisation ", Revue française de science politique, vol. 54, nº ${ }^{\circ}$, p. 877-909.

Ferrand-Bechmann D. (2011), Les bénévoles face au cancer, Paris, Desclée de Brouwer.

Fillion E. (2009), À l'épreuve du sang contaminé. Pour une sociologie des affaires médicales, Paris, Éditions de l'École des Hautes Études en Sciences Sociales.

Herzlich C. (1995), "Les difficultés de constitution d'une cause», Sciences sociales et santé, vol. $13, \mathrm{n}^{\circ} 4$, p. 39-44.

Ion J. (1997), La fin des militants ?, Paris, Éditions de l'Atelier.

Kivits J. (2012), "Les usages de l'Internet-santé », in Thoër C., Lévy J.J. (dir.), Internet et santé. Acteurs, usages et appropriations, Sainte-Foix, Presses de l'Université du Québec, p. 37-56.

Knobé S. (2007), Le besoin d'inscription dans des collectifs de patients atteints de cancerparticipation associative, structuration des collectifs de malades, Rapport de postdoctorat pour l'Institut national du cancer.

- (2009), «Logiques d'engagement des malades dans les associations de lutte contre le cancer», Socio-logos. Revue de l'association française de sociologie, ${ }^{\circ} 4$. URL: http://socio-logos.revues.org/2346

- (2010), "À propos des collectifs d'usagers de la santé en France au 20 ème siècle », Journal of global health promotion, vol. 17, $\mathrm{n}^{\circ} 3$, p. 85-88.

Knobé S., Briatte F. (2010), « Institutionnalisation et médiatisation d'une prise de parole collective chez les malades atteints de cancer : les "États généraux" de la Ligue nationale contre le cancer», in H. Romeyer (dir), La santé dans l'espace public, Éditions EHESP, Rennes, p. 159-173.

La Ligue nationale contre le cancer (1999), Les malades prennent la parole. Le livre blanc des $1^{\text {ers }}$ États Généraux des malades du cancer, Paris, Ramsay.

Legros M. (2009), «Étude exploratoire sur les blogs personnels santé et maladie », Santé publique, vol. 21, HS nov.-dec., p. 41-51.

Ménoret M. (1999), Les temps du cancer, Paris, Éditions du CNRS. 